\title{
Status of the semileptonic $B$ decays and muon g-2 in a Type-III 2HDM with right-handed neutrinos
}

\section{Syuhei Iguro*}

Department of Physics, Nagoya University, Nagoya 464-8602, Japan

E-mail: iqurodeken.phys.nagoya-u.ac.jp

In this work, we study the extended Standard Model (SM) with an extra Higgs doublet and righthanded neutrinos. If the symmetry to distinguish the two Higgs doublets is not assigned, flavor changing neutral currents (FCNCs) involving the scalars are predicted even at the tree level. We investigate the constraints on the FCNCs at the one-loop level, and especially study the semileptonic $B$ meson decays, e.g. $B \rightarrow D^{(*)} \tau \nu$ and $B \rightarrow K^{(*)} l l$ processes, where the SM predictions are more than $2 \sigma$ away from the experimental results. We also consider the flavor-violating couplings involving right-handed neutrinos and discuss if the parameters to explain the excesses of the semileptonic $B$ decays can resolve the discrepancy in the anomalous muon magnetic moment. Based on the analysis, we propose the smoking-gun signal of our model at the LHC.

The 39th International Conference on High Energy Physics (ICHEP2018)

4-11 July, 2018

Seoul, Korea

${ }^{*}$ Speaker. 


\section{Introduction}

Recently, the Type-III Two Higgs Doublet Model(2HDM) is attracting a lot of attention, since it is one of the good candidates to accomodate the excesses reported by the BaBar, Belle, and LHCb collaborations. The semileptonic $B$ decays, $B \rightarrow D^{(*)} \tau \nu$, have been measured and their results deviate from the SM predictions [四]. Although it is found that the explanation of $B \rightarrow D^{*} \tau \nu$ contradicts the leptonic $B_{c}$ decay [ [ $[$ ] and the $\tau v$ resonance search in the LHC [B]], the Type-III 2HDM is still

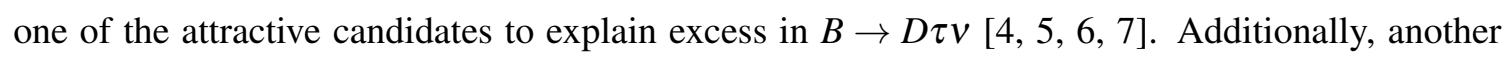
semileptonic $B$ decay, i.e. $B \rightarrow K^{(*)} \mu \mu$, is also discussed recently in the 2HDM [ [ 8$]$ ]. In this process, the $\mathrm{LHCb}$ collaboration has reported the deviations from the SM predictions in the measurements concerned with the angular observables [0] and the lepton universality [ए0]. Moreover, it is known that the Type-III 2HDM can accomplish the explanation of the anomalous muon magnetic moment $\left((g-2)_{\mu}\right)$ deviated from the SM prediction [ए]]. In this work we summarized the possibility of simultaneous explanation of them and studied an implication for the collider physics.

\section{Model:Type-III 2HDM}

We introduce the so-called Type-III 2HDM with right-handed neutrinos. There are two Higgs doublets in our model. We take the Higgs basis in which only one Higgs doublet obtains the nonzero VEV, the fields can be decomposed as [12]

$$
H_{1}=\left(\begin{array}{c}
G^{+} \\
\frac{v+\phi_{1}+i G}{\sqrt{2}}
\end{array}\right), \quad H_{2}=\left(\begin{array}{c}
H^{+} \\
\frac{\phi_{2}+i A}{\sqrt{2}}
\end{array}\right),
$$

where $G^{+}$and $G$ are Nambu-Goldstone bosons, and $H^{+}$and $A$ are a charged Higgs boson and a CPodd Higgs boson, respectively. $v$ is the VEV: $v \simeq 246 \mathrm{GeV}$. In this base, we write down the Yukawa couplings with the SM fermions. In the mass basis of the fermions, the Yukawa interactions are given by

$$
\begin{aligned}
\mathscr{L}= & -\bar{Q}_{L}^{i} H_{1} y_{d}^{i} d_{R}^{i}-\bar{Q}_{L}^{i} H_{2} \rho_{d}^{i j} d_{R}^{j}-\bar{Q}_{L}^{i}\left(V^{\dagger}\right)^{i j} \widetilde{H}_{1} y_{u}^{j} u_{R}^{j}-\bar{Q}_{L}^{i}\left(V^{\dagger}\right)^{i j} \widetilde{H}_{2} \rho_{u}^{j k} u_{R}^{k} \\
& -\bar{L}_{L}^{i} H_{1} y_{e}^{i} e_{R}^{i}-\bar{L}_{L}^{i} H_{2} \rho_{e}^{i j} e_{R}^{j}-\bar{L}_{L}^{i}\left(V_{v}\right)^{i j} \widetilde{H}_{1} y_{v}^{j} v_{R}^{i}-\bar{L}_{L}^{i}\left(V_{v}\right)^{i j} \widetilde{H}_{2} \rho_{v}^{j k} v_{R}^{k},
\end{aligned}
$$

where $i, j$ and $k$ are flavor indices, and $Q=\left(V^{\dagger} u_{L}, d_{L}\right)^{T}, L_{L}=\left(V_{v} v_{L}, e_{L}\right)^{T}$ are defined. $\widetilde{H}_{1,2}$ denote $\widetilde{H}_{1,2}=i \tau_{2} H_{1,2}^{*}$, where $\tau_{2}$ is the Pauli matrix. $V$ is the CKM matrix and $V_{v}$ is the MNS matrix. Fermions $\left(f_{L}, f_{R}\right)(f=u, d, e, v)$ are mass eigenstates, and $y_{i}^{f}=\sqrt{2} m_{f_{i}} / v$, where $m_{f_{i}}$ are the fermion masses.. $\rho_{f}^{i j}$ are the Yukawa couplings that are independent of the SM fermion mass matrices.

There are three types of the scalars: the charged Higgs $\left(H^{ \pm}\right)$, the CP-odd scalar $(A)$ and the two CP-even scalars $\left(\phi_{1,2}\right)$. The CP-even scalars are not mass eigenstates, although the mixing should be tiny not to disturb the SM prediction. The mixing is defined as

$$
\left(\begin{array}{c}
\phi_{1} \\
\phi_{2}
\end{array}\right)=\left(\begin{array}{cc}
\cos \theta_{\beta \alpha} & \sin \theta_{\beta \alpha} \\
-\sin \theta_{\beta \alpha} & \cos \theta_{\beta \alpha}
\end{array}\right)\left(\begin{array}{c}
h \\
H
\end{array}\right) .
$$

$\rho_{f}$ are $3 \times 3$ matrices and the each element is the free parameter that is constrained by the flavor physics and the collider experiments. The comprehensive study about the phenomenology in the 
Type-III 2HDM has been done in Ref. [ㅍ]]. However there are many choices for the matrix alignment, actually only a few elements or combinations of them are allowed to be sizable according to the stringent experimental bounds. The all off-diagonal elements of $\rho_{d}$ are strongly constrained by the $\Delta F=2$ processes. $\rho_{u}^{u c}$ and $\rho_{u}^{c u}$ have to be small to escape from the stringent constraint that given by the $D-\bar{D}$ mixing. Moreover, $\rho_{u}^{u t}$ and $\rho_{u}^{t u}$ are severely constrained by the $K-\bar{K}$ mixing at the one-loop level. Thus, it is difficult to expect that the couplings between the light quarks $(u, d, s)$ and the other quarks are larger than $\mathscr{O}(0.01)$. Based on the examination, we consider the case that $\left|\rho_{u}^{c t}\right|$ and/or $\left|\rho_{u}^{t c}\right|$ are sizable. One of our motivations of this study is to investigate the compatibility among the explanations of the excesses in the Type-III 2HDM. It is pointed out that the sizable $\rho_{u}^{t c}$ can improve the discrepancies in the $b \rightarrow s l l$ and $b \rightarrow c l v$ processes [焑].

We can also find the strong upper bounds on the Yukawa couplings in the lepton sector. The lepton flavor violating (LFV) processes are predicted by the neutral scalar exchanging, if the offdiagonal elements of $\rho_{e}$ are sizable. If the extra Yukawa couplings involving electron are large, the LEP experiment can easily exclude our model. Interestingly, the authors of Ref. [W] have pointed out that the large $\rho_{e}^{\mu \tau} \times \rho_{e}^{\tau \mu}$ can achieve the explanation of the $(g-2)_{\mu}$, that is largely deviated from the SM prediction. The explanation, however, requires the other Yukawa couplings to be small [Ш]]. Eventually, we consider the following simple textures of $\rho_{f}$ from the phenomenological point of view:

$$
\rho_{u} \simeq\left(\begin{array}{ccc}
0 & 0 & 0 \\
0 & 0 & \rho_{u}^{c t} \\
0 & \rho_{u}^{t c} & \rho_{u}^{t t}
\end{array}\right),\left|\rho_{d}^{i j}\right| \ll \mathscr{O}(0.1), \rho_{e} \simeq\left(\begin{array}{ccc}
0 & 0 & 0 \\
0 & 0 & \rho_{e}^{\mu \tau} \\
0 & \rho_{e}^{\tau \mu} & 0
\end{array}\right)
$$

In our study, we also consider the contribution of $\rho_{v}$ to flavor physics. This investigation has not been done well in the type-III 2HDM. This is because the tiny Dirac neutrino masses predict small Yukawa couplings so that $\rho_{v}$ is also naively expected to be small. $\rho_{v}$, however, does not contribute to the active neutrino masses, directly. If both $\rho_{v}$ and $\rho_{e}$ are sizable, $\rho_{v}$ would contribute to the neutrino masses radiatively. Otherwise, $\rho_{v}$ could be large compared to $y_{v}^{i}$, in the bottomup approach. The unique texture as in Eq. (2.4) may also allow $\rho_{v}$ to be sizable. Based on this consideration, we study the upper bound on $\rho_{v}$ and discuss the impact on the flavor physics.

\section{The possibility of simultaneous explanation}

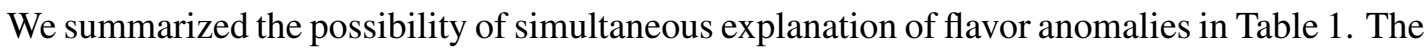
Dirac neutrino case predicts $N_{e f f} \approx 4$ and is in tension with the recent cosmological observation. The neutrino trident production also excludes the case with $\rho_{v}>1$. We can also introduce the small Majorana mass term, $m_{R}$, to decrease $N_{\text {eff }}$.

\section{Implication for the collider physics}

In order to explain $R(D)$ and muon g-2 simultaneously, $\rho_{u}^{t c}$ needs to be $\mathscr{O}(1)$. Then the samesign top signal is predicted. If the neutral scalars, $H$ and $A$, are not degenerate, the same-sign top signal, $p p \rightarrow t t$, is enhanced by $\rho_{u}^{c t}, \rho_{u}^{t c}$ couplings. The current upper bound on the cross section is $1.2 \mathrm{pb}$ at the LHC with $\sqrt{s}=13 \mathrm{TeV}$ [प]4]. When $m_{A}=200 \mathrm{GeV}$ and $m_{H}=250 \mathrm{GeV}$, the each 


\begin{tabular}{|c|c|c|c|c|c|}
\hline & $R\left(K^{(*)}\right)$ & $P_{5}^{\prime}$ & $R(D)$ & $R\left(D^{*}\right)$ & $\delta \alpha_{\mu}$ \\
\hline \hline \multicolumn{7}{|c|}{$(\mathrm{B}) \rho_{e} \neq 0, \rho_{v}=0$} \\
\hline \hline$\rho_{u}^{t t}$ & $\times$ & $\times$ & $\times$ & $\times$ & $\bigcirc$ \\
\hline$\rho_{u}^{t c}$ & $\times$ & $\bigcirc$ & $\bigcirc$ & $\times$ & $\times$ \\
\hline$\rho_{u}^{c t}$ & $\times$ & $\times$ & $\times$ & $\times$ & $\bigcirc$ \\
\hline \hline \multicolumn{7}{|c|}{$(\mathrm{C}) \rho_{e}=0, \rho_{v} \neq 0$} \\
\hline \hline$\rho_{u}^{t t}$ & $\triangle$ & $\triangle$ & $\times$ & $\times$ & $\times$ \\
\hline$\rho_{u}^{t c}$ & $\times$ & $\bigcirc$ & $\bigcirc$ & $\times$ & $\times$ \\
\hline$\rho_{u}^{c t}$ & $\triangle$ & $\triangle$ & $\times$ & $\times$ & $\times$ \\
\hline
\end{tabular}

Table 1: Summary of the capabilities to explain the excesses. In the each observable, our prediction is evaluated by the symbols, " $\bigcirc$ ", " $\triangle$ " and " $\times$ ". The symbol, " $\bigcirc$ ", means that our predictions are within the $1 \sigma$ regions of the experimental results. In the box with " $\times$ ", our prediction is out of the $2 \sigma$ region. In the box with " $\triangle$ ", the predictions can be within the $2 \sigma$ region of the experimental results, i.e., $P_{5}^{\prime}$ and $R(K)=0.745_{-0.074}^{+0.090} \pm 0.036 \simeq 0.745_{-0.082}^{+0.097}\left(q^{2}[1,6] \mathrm{GeV}^{2}\right)[$ [ᄄ口] $]$, if $\rho_{v}$ is $\mathscr{O}(1)$.

cross section is estimated as

$$
\begin{aligned}
\sigma(p p \rightarrow t t+\overline{t t}) & =4.23 \times 10^{-3}\left|\rho_{u}^{t c}\right|^{4}[\mathrm{pb}], \\
\sigma(p p \rightarrow t t \bar{c}+\bar{t} c) & =4.13 \times 10^{-1}\left|\rho_{u}^{t c}\right|^{4}[\mathrm{pb}], \\
\sigma(p p \rightarrow t t \bar{c} \bar{c}+\bar{t} \bar{t} c c) & =1.14 \times 10^{-1}\left|\rho_{u}^{t c}\right|^{4}[\mathrm{pb}] .
\end{aligned}
$$

However our prediction is below the experimental bound, HL-LHC may test an interesting scenario in the future. Other signals e.g. $p p \rightarrow \bar{b} H^{-} \rightarrow \bar{b} \tau \bar{v}, p p \rightarrow \bar{t} H^{+} \rightarrow \bar{t} \tau \bar{v}$ are also studied in [[], 团].

\section{References}

[1] Y. Amhis et al. [HFLAV Collaboration], Eur. Phys. J. C 77, no. 12, 895 (2017)

[2] R. Alonso, B. Grinstein and J. Martin Camalich, Phys. Rev. Lett. 118, no. 8, 081802 (2017)

[3] S. Iguro, Y. Omura and M. Takeuchi, arXiv:1810.05843 [hep-ph].

[4] A. Crivellin, C. Greub and A. Kokulu, Phys. Rev. D 86, 054014 (2012)

[5] M. Tanaka and R. Watanabe, Phys. Rev. D 87, no. 3, 034028 (2013)

[6] S. Iguro and K. Tobe, Nucl. Phys. B 925, 560 (2017)

[7] S. Iguro and Y. Omura, JHEP 1805, 173 (2018)

[8] Q. Y. Hu, X. Q. Li and Y. D. Yang, Eur. Phys. J. C 77, no. 3, 190 (2017)

[9] R. Aaij et al. [LHCb Collaboration], Phys. Rev. Lett. 111, 191801 (2013)

[10] R. Aaij et al. [LHCb Collaboration], Phys. Rev. Lett. 113, 151601 (2014)

[11] Y. Omura, E. Senaha and K. Tobe, JHEP 1505, 028 (2015)

[12] S. Davidson and H. E. Haber, Phys. Rev. D 72, 035004 (2005)

[13] A. Crivellin, A. Kokulu and C. Greub, Phys. Rev. D 87, no. 9, 094031 (2013)

[14] A. M. Sirunyan et al. [CMS Collaboration], Eur. Phys. J. C 77, no. 9, 578 (2017) 